\title{
Retention of Metal Crowns Luted to Implant Abutments of Two Different Diameters using Different Luting Agents
}

\author{
Jay Dipak Matani, Natasha Parulekar, Mohit Kheur, Shantanu Jambhekar, MK Supriya
}

\begin{abstract}
Purpose: The desirable properties of a luting agent used for implant prostheses are adequate tensile strength for retention and at the same time render the crowns to be retrievable. No studies have reported a comparison of the retention of implant crowns luted with zinc phosphate and polymeric cements. Studying the retentive abilities of these cements utilizing varying sizes of implant abutments may provide new insights into their clinical behaviors.
\end{abstract}

Aim: To compare the retentive abilities of two luting agents, using them to lute crowns on implant abutments of different diameters.

Materials and methods: Two implants $(3.7$ and $6.0 \mathrm{~mm}$ diameter) were embedded in a resin block. Straight abutments of corresponding diameters were screwed into the implants. The height of abutments was kept equal for both abutments $(8 \mathrm{~mm})$. Seven copings were fabricated for each abutment. Groups 1 and 2 copings ( 14 copings) were tested for both luting agents; zinc phosphate and polymeric cement using a universal testing machine.

Results: For the abutment diameters tested, the retentive values of zinc phosphate was significantly greater than polymeric implant cement. For the broad abutment, zinc phosphate was significantly more retentive than polymeric implant cement. For the narrow abutment, retentive values of zinc phosphate and polymeric implant cement were almost similar.

Conclusion: The retentive values of zinc phosphate were significantly greater than polymeric implant cement. For broad abutment, zinc phosphate was more retentive whereas for narrow abutment, retentive values were almost similar.

Keywords: Cemented implant restorations, Dental cements, Dental implants.

How to cite this article: Matani J D, Parulekar N, Kheur M, J ambhekar S, Supriya MK. R etention of Metal Crowns Luted to Implant Abutments of Two Different Diameters using Different Luting Agents. IntJ Prosthodont Restor Dent 2012;2(3):96-100.

Source of support: Nil

Conflict of interest: None

\section{INTRODUCTION}

Introduction of osseointegrated implants has brought about a great change in the treatment of partially and completely edentulous patients. Restorations supported by implants can be either cement retained or screw retained. ${ }^{1}$ Cementretained restorations have gained preference in many cases due to inherent advantages, ${ }^{2}$ making them the restorations of choice for the treatment of implant patients.

$V$ arious luting agents likezinc phosphate, glass ionomer cement, among the many others have been routinely used for the purpose of luting restorations. ${ }^{3}$ Zinc phosphate serves as a gold standard for luting of implant supported restorations. However, its higher retentive property proves to be a draw back while trying to retrieve the restorations. ${ }^{4}$

Polymeric implant cements are used now-a-days as they are adequately retentive and at the same time have good retrievability. Data reported on the bond strength for provisional luting agents with castings cemented to implant abutments is limited. ${ }^{5}$

Different clinical situations demand the need of different diameter abutments. 0 ne of the key factors for retention of restorations is the surface area of the abutment. ${ }^{6} \mathrm{H}$ ow ever, the effect of increase in surface area (wider abutments) contributing to retention has not been documented.

The purpose of this study is to compare the retention of metal crowns to implant abutments of two different diameters using two different luting agents.

\section{MATERIALS AND METHODS}

A clinical situation was simulated by fabricating two identical clear autopolymerizing acrylic resin blocks into each of which an implant, one of $3.7 \mathrm{~mm}$ in diameter (Group 1) (Uniti Implants, Equinox M edical Technology, A mersfoort, N etherlands) and other of $6.0 \mathrm{~mm}$ in diameter (Group 2) (Uniti Implants, Equinox M edical Technology, A mersfoort, N etherlands) were embedded in the center.

Straight abutments of the corresponding diameter, each of a $2 \mathrm{~mm}$ collar height and abutment height of $8 \mathrm{~mm}$ were placed into the implants and the screw was torqued up to $23 \mathrm{~N} \mathrm{~cm}$ torque. The access holes of these screws were blocked out using Fermit resin (Ivoclar Vivadent, Ivoclar Vivadent AG, Liechtenstein) (Figs 1 and 2).

\section{Coping Fabrication}

The copings were waxed up using type II inlay casting wax (S-U-M odelling wax, Schuler Dental G mbH and Co. K G, U Im, Germany) and were cast in nickel-chromium alloy (Hi-Chrom Soft-7, High-D ental, Japan) as per standard casting protocols.

Each coping was $0.5 \mathrm{~mm}$ thick and had a circular loop $5 \mathrm{~mm}$ in diameter on its occlusal surface to permit the attachment of the hook of the testing machine to it.

A total of 14 copings were fabricated (seven copings for each study group) (Figs 3 and 4). 


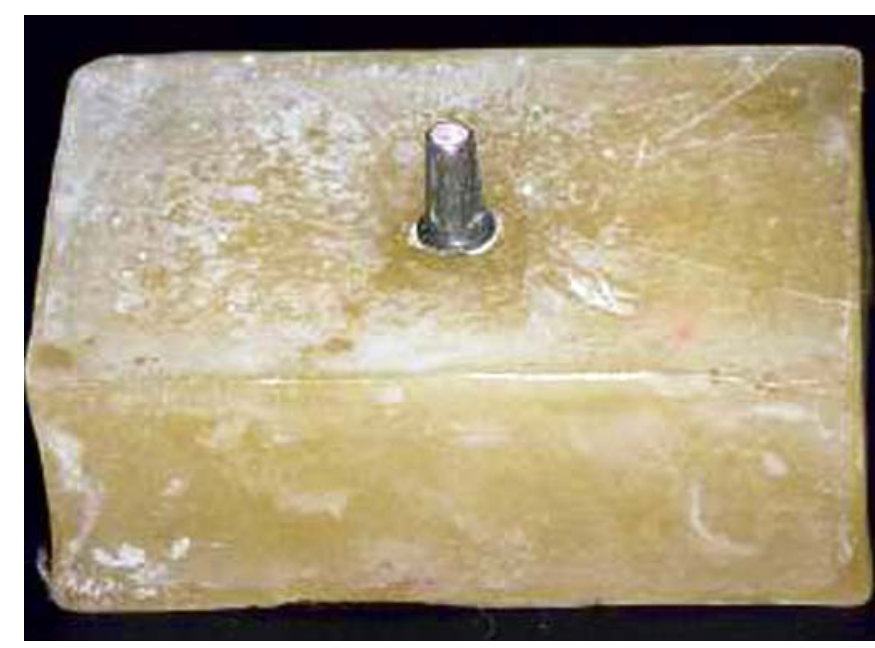

Fig. 1: A $3.7 \mathrm{~mm}$ diameter straight abutment (group 1)

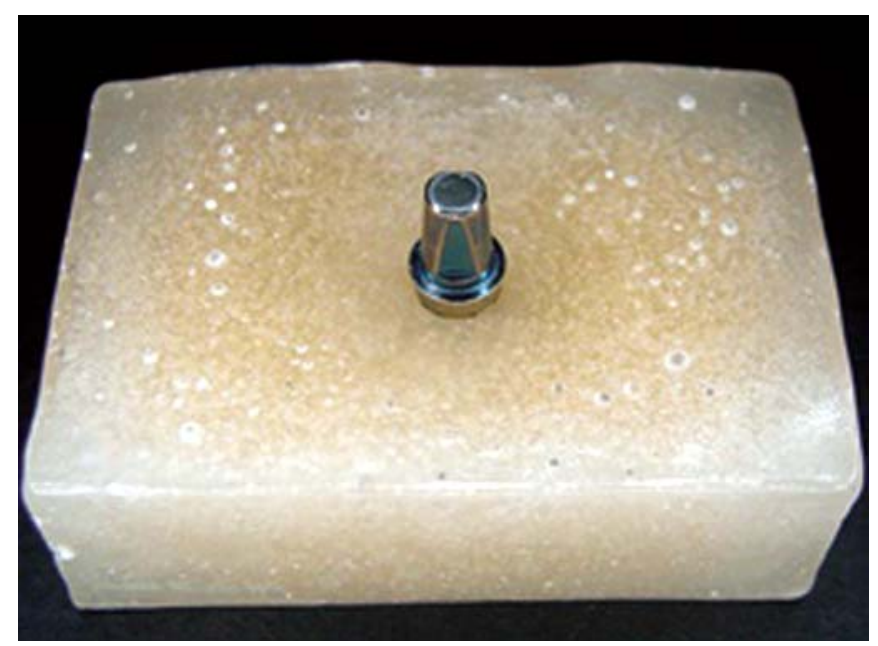

Fig. 2: A $6 \mathrm{~mm}$ diameter straight abutment (group 2)

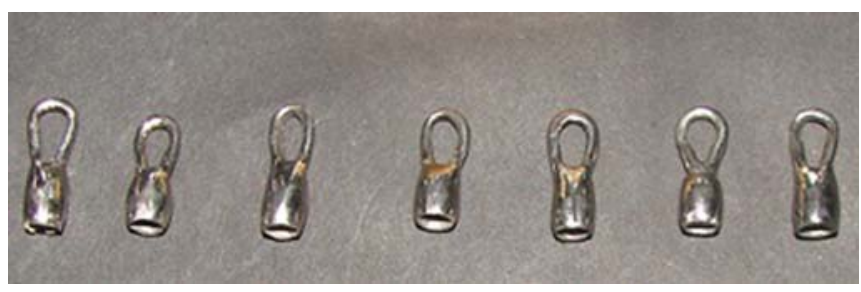

Fig. 3: Copings in group 1

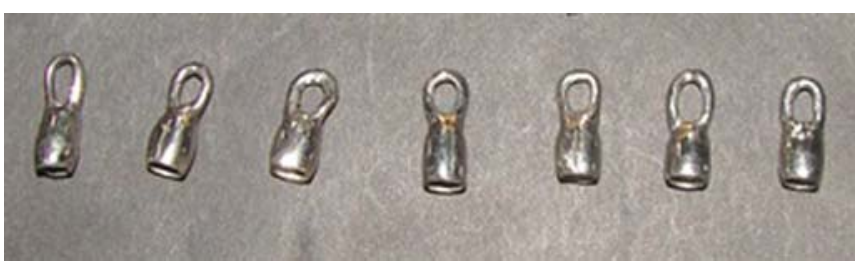

Fig. 4: Copings in group 2

\section{Cementation of Copings}

Prior to cementation the copings were sand-blasted using 50 micron alumina powder. Two luting cements, zinc

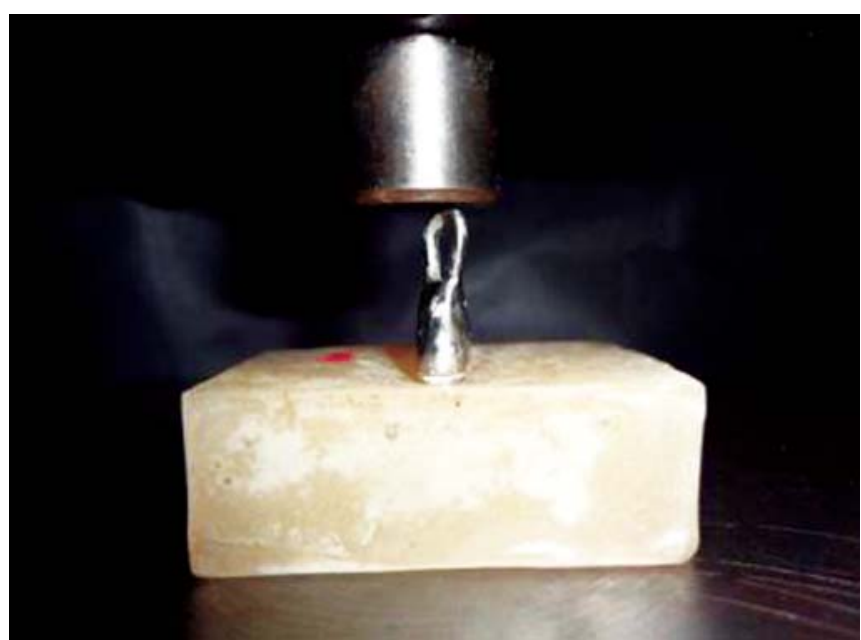

Fig. 5: Cementation of coping under load of $100 \mathrm{~N}$

phosphate cement (H arvard, H arvard Dental International, B erlin, Germany) and polymeric resin cement (Implacem, Equinox M edical Technology, A mersfoort, Netherlands) were used for luting the copings to the abutments (Fig. 5). For each group the sequence in which the two luting agents were used was randomly selected.

\section{Luting Procedure with Zinc Phosphate Luting Agent}

The powder and liquid were weighed using a digital balance in a ratio recommended by manufacturer. The mixing was done on a cool clean and dry glass slab. The luting agent was then coated on the inner side of the coping and the coping were placed on the respective abutment. The blocks were clamped onto a loading device and the pressure on the coping was gradually increased up to $100 \mathrm{~N}$. A fter a setting time of 4 minutes, the excess cement was removed with a probe. The block was then stored overnight at room temperature.

\section{Luting Procedure with Polymeric Implant Cement (ImplaCem)}

The luting agent is delivered in an automix form. The injection tip was attached to the cement cartridge and the cement was extruded into each coping. The coping was then placed on the abutment and the blocks were similarly clamped onto the loading device so that the copings were cemented under a constant $100 \mathrm{~N}$ load as described earlier.

Each coping was luted with any one luting agent initially, and then was subjected to testing after 24 hours. Following testing, the copings were manually and ultrasonically cleaned and were luted back on the same abutment with the other luting agent. The purpose of this was to ensure that the same coping was tested with both luting agents (Fig. 6). 


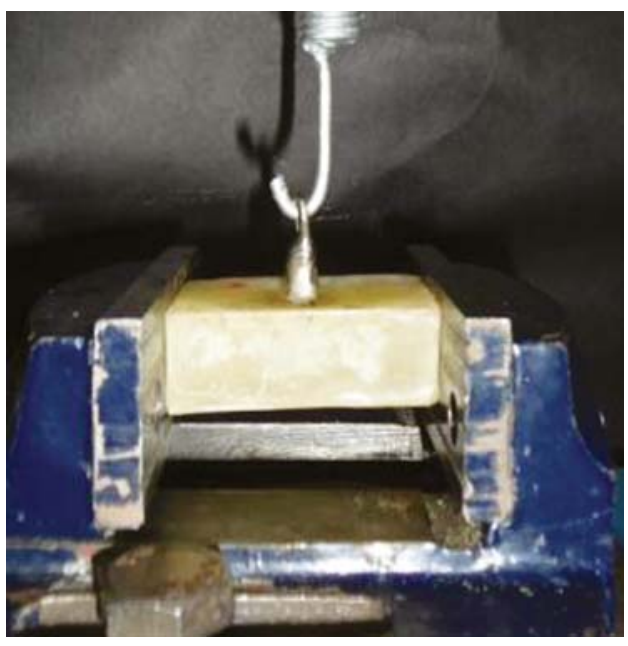

Fig. 6: Testing of the coping

\section{Testing of Specimens}

The blocks with the coping cemented on them were locked on a universal testing machine. The hook of the testing device was engaged into the loop created on the occlusal surface of the coping and a load was applied to pull the coping away from the abutment at a speed of $5 \mathrm{~mm} /$ minute. The force at which the bond failure occurred was recorded as the peak load of the failure of the luting cement.

All the samples were subjected to the same testing protocols for both the luting cements.

\section{STATISTICAL ANALYSIS}

Unpaired t-test was used to compare the load at which failure occurred between the two cements for the narrow abutment and broad abutments respectively.

Similarly comparison was made betw een the narrow and broad abutment for the zinc phosphate group and polymeric cement group respectively using unpaired t-test. O ne-way analysis of variance (A N OVA) and ad-hoc test for multiple comparisons between various groups was utilized, $\mathrm{p}$-values were obtained for all the tests. V alue less than 0.05 are considered as significant and value more than 0.05 are not significant. The entire statistical analysis was done using Statistical Package for Social Sciences (SPSS Inc. Chicago, USA, version 11.5) for M icrosoft Windows.

\section{RESULTS}

The load required for failure for both the groups were recorded and the mean and standard deviations were tabulated (Table 1). For both the abutments zinc phosphate show ed superior retention than polymeric implant cements (Graph 1). A significant difference was present between the groups (Table 2).

In this study, for the broad abutment (6 mm diameter), it was found that there was a significant difference in the retentive values obtained by using zinc phosphate cement (mean $=667.370$ ) when compared to those of the polymeric implant cement ( mean $=256.55)(p=0.000)($ Table 3$)$.

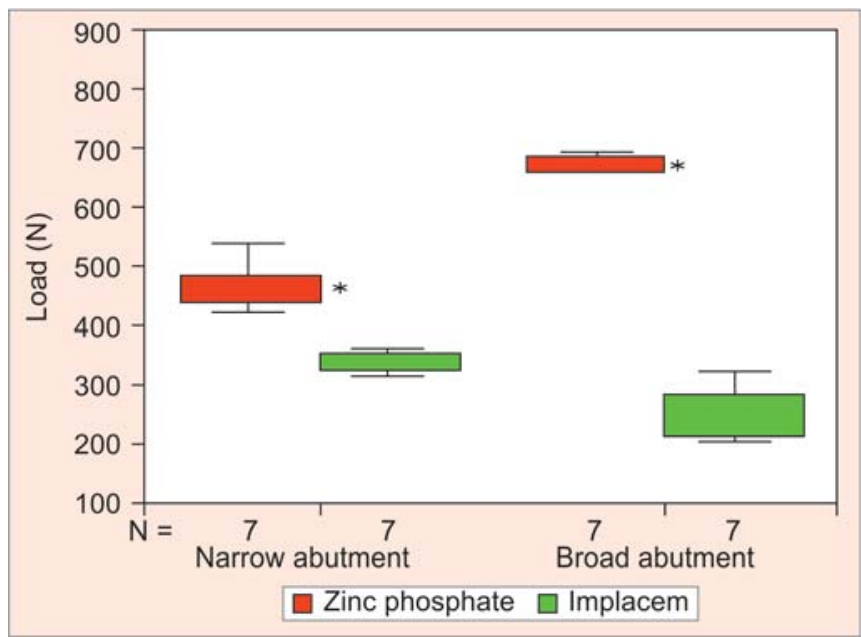

Graph 1: Comparison of load at failure for groups 1 and 2

\begin{tabular}{lllrrrr} 
& \multicolumn{2}{c}{ Table 1: Load at failure for different groups } \\
& N & Mean & Std. deviation & Std. error & \multicolumn{2}{c}{ 95\% confidence interval for mean } \\
\cline { 3 - 6 } & & & & & Lower bound & Upper bound \\
\hline Group 1 ZP & 7 & 464.2 & 41.9 & 15.8 & 425.5 & 503.0 \\
Group 1 PIC & 7 & 337.8 & 17.9 & 6.8 & 321.2 & 354.4 \\
Group 2 ZP & 7 & 667.4 & 67.6 & 25.6 & 604.8 & 729.9 \\
Group 2 PIC & 7 & 256.6 & 46.2 & 17.5 & 213.8 & 299.3 \\
\hline
\end{tabular}

ZP: Zinc phosphate cement; PIC: Polymeric implant cement

Table 2: Results for ANOVA

\begin{tabular}{lccccc}
\hline & Sum of squares & df & Mean square & F & Significance \\
\hline Between groups & $672,635.151$ & 3 & $224,211.717$ & 102.060 & 0.000 \\
Within groups & $52,724.651$ & 24 & $2,196.860$ & & \\
\hline Total & $725,359.802$ & 27 & & & \\
\hline
\end{tabular}


Retention of Metal Crowns Luted to Implant Abutments of Two Different Diameters using Different Luting Agents

\begin{tabular}{|c|c|c|c|c|c|c|}
\hline \multirow[t]{2}{*}{ Category (I) } & \multirow[t]{2}{*}{ Category (J) } & \multirow{2}{*}{$\begin{array}{l}\text { Mean difference } \\
(\mathrm{I}-\mathrm{J})\end{array}$} & \multirow[t]{2}{*}{ Std. error } & \multirow[t]{2}{*}{ Significance } & \multicolumn{2}{|c|}{$95 \%$ confidence interval } \\
\hline & & & & & Lower bound & Upper bound \\
\hline \multirow[t]{3}{*}{ ZP 1} & PIC 1 & 126.4 & 25.05 & 0.000 & 57.3 & 195.5 \\
\hline & ZP 2 & -2.03 & 25.05 & 0.000 & -272.3 & -134.0 \\
\hline & PIC 2 & 207.7 & 25.05 & 0.000 & 138.6 & 276.8 \\
\hline \multirow[t]{3}{*}{ PIC 1} & ZP 1 & -126.41 & 25.05 & 0.000 & -195.5 & -57.3 \\
\hline & ZP 2 & -329.56 & 25.05 & 0.000 & -398.7 & -260.4 \\
\hline & PIC 2 & 81.3 & 25.05 & 0.017 & 12.2 & 150.4 \\
\hline \multirow[t]{3}{*}{ ZP 2} & ZP 1 & 203.1 & 25.05 & 0.000 & 134.0 & 272.3 \\
\hline & PIC 1 & 329.6 & 25.0 & 0.000 & 260.4 & 398.7 \\
\hline & PIC 2 & 410.8 & 25.0 & 0.000 & 341.7 & 479.9 \\
\hline \multirow[t]{3}{*}{ PIC 2} & ZP 1 & -207.68 & 25.0 & 0.000 & -276.8 & -138.6 \\
\hline & PIC 1 & -81.3 & 25.05 & 0.017 & -150.4 & -12.2 \\
\hline & ZP 2 & -410.82 & 25.0 & 0.000 & -479.9 & -341.7 \\
\hline
\end{tabular}

ZP 1: Group 1 zinc phosphate cement; PIC 1: Group 1 polymeric implant cement; ZP 2: Group 2 zinc phosphate cement; PIC 2: Group 2 polymeric implant cement

However, when the narrow abutment ( $3.7 \mathrm{~mm}$ diameter) was used, the difference in the retentive value of zinc phosphate (mean $=464.228$ ) and polymeric implant cement (mean $=337.814$ ) was not as much as seen for the broad abutment. This difference though was statistically significant $(p=0.000)$ (Table 3).

\section{DISCUSSION}

Cement retained implant restorations are the mainstay of modern implantology. The choice of luting agent may directly affect the long-term success of the implant restorations. ${ }^{3,7-9}$

Cemented restorations have become a popular al ternative and exhibit the following advantages over screwretained restorations:

a. Provide resistance to rotational forces that cause loosening, as in screw-retained designs.

b. Provide passive superstructure and allow easier axial loading of fixture.

c. They are less technique sensitive and hence allow completion of laboratory and clinical phases of restorative treatment with ease.

d. Small discrepancies between retainer and abutment can be compensated. ${ }^{10}$

e. Easier access on posterior abutments.

The amount of retention and resistance form of cement retained restorations depends on the selection of the abutment, luting agent and casting procedures. ${ }^{11}$

Some of the factors affecting the same are the use of venting or internal casting relief, variations in cement viscosity and seating pressure and the masticatory forces.

L uting considerations for implant supported crowns differ slightly from those for tooth supported crowns and depend on the type of prosthesis and the long-term goals.

Desirable properties of a luting agent used for luting implant supported crowns are: a. A dequate tensile strength to provide good retention and at the same time render the crowns to be retrievable without damaging the superstructure/abutment.

b. Basic requirements like mechanical, biological and handling properties must be met. However, fluoride releasing and bonding to tooth is not needed in the case of implant supported restorations.

c. Easy and predictable cleaning of the excess residual cement from the abutment surface and peri-implant sulcus.

Zinc phosphate is the most commonly used luting agent for restorations. It has been reported that the use of zinc phosphate cement results in significantly higher load separation values when compared to Tempbond NE (Kerr $\mathrm{M}$ anufacturing $\mathrm{Co}$. Romulus M I). ${ }^{12} \mathrm{H}$ owever, its strength is quite high to render easy retrievability and removal of excess cement from the gingival sulcus is frequently a problem.

For ease of removal, temporary luting agents are mainly used for luting implant supported restorations. With the current trend of using temporary cements, each advantage of screw retained FPD can be applied to the cemented restoration as well. ${ }^{13} \mathrm{~N}$ ew er cements like Implacem which are polymeric by nature are gaining popularity due to the significant retentive capability and ease in cleaning excess residual cement from the peri-implant sulcus. Hence, zinc phosphate and polymeric implant cement were selected for comparison of their retentive abilities.

As stated previously, the abutment size plays a critical role in the retention of the implant supported prosthesis. Previous studies have reported the effect of abutment size (diameter and height) on the retentiveness of the prosthesis but no clear conclusion has been drawn. ${ }^{9,14}$ Thus, studying the retentive abilities of these cements on implant abutments of varying sizes (diameter) may provide some new insights into their behavior and clinical applications. 
Zinc phosphate showed superior retention than polymeric implant cements for both the abutment diameters (Graph 1).

In this study, for the broad abutment (6 $\mathrm{mm}$ diameter), it was found that retentive values obtained by using zinc phosphate cement were significantly greater than polymeric implant cement (Implacem). The values obtained using the polymeric implant cement are close to those of the average masticatory forces thus indicating that polymeric implant cements should not be used for broader abutments to prevent loss of retention of the implant prostheses.

However, the difference in the retentive value of zinc phosphate and polymeric implant cement (Implacem) for the narrow abutment ( $3.7 \mathrm{~mm}$ diameter) was not as much as seen for the broad abutment. This difference though was statistically significant (Table 3). As I mplacem provides retentive values almost similar to that of zinc phosphate, Implacem maybe preferred for such narrower abutments, due to its ease of removal from the sulcus and retrievability. The simplicity of manipulation by the use of its automated dispensing tips is also a potential advantage.

\section{CONCLUSION}

W ithin the limitations of the study the following conclusions can be drawn:

- For the abutment diameters tested, the retentive values of zinc phosphate was significantly greater than polymeric implant cement.

- For broad abutments, zinc phosphate was significantly more retentive than polymeric implant cement.

- For narrow abutments, retentive values of zinc phosphate and polymeric implant cement were almost similar.

\section{CLINICAL SIGNIFICANCE}

The following clinical recommendations can be drawn,

For broader abutments, zinc phosphate should be preferred over the polymeric cements.

For narrower abutments, as the retentive values of both the luting cements are in the similar range, due to the inherent advantages of the polymeric cement, it could be the material of choice.

\section{REFERENCES}

1. M ichalakis KX, Hirayama H, Garefis PD. Cement-retained versus screw-retained implant restorations: A critical review. Int J Oral M axillofac Implants 2003;18:719-28.

2. Jemt $T$, L indén $B$, Lekholm U. Failures and complications in 127 consecutively placed fixed partial prostheses supported by B rånemark implants: From prosthetic treatment to first annual check-up. Int J Oral M axillofac Implants 1992;7:40-44.
3. Squier RS, A gar JR, Duncan JP, Taylor TD. Retentiveness of dental cements used with metallic implant components. Int J Oral M axillofac Implant 2001;16:793-98.

4. Clayton GH, D riscoll CF, H ondrum SO. The effect of luting agents on the retention and marginal adaptation of the CeraO ne implant system. Int J Oral M axillofac Implants 1997;12:660-65.

5. B reeding $L C, D$ ixon $D L, B$ ogacki $M T$, Tietge JD. U se of luting agents with an implant system: Part I. J Prosthet Dent 1992;68:737-41.

6. Kaufman EG, Coelho DH, Colin L. Factors influencing the retention of cemented gold castings. J Prosthet Dent 1961;11(3):487-502.

7. Kerby RE, McGlumphy EA, Holloway JA. Some physical properties of implant abutment luting cements. Int] Prosthodont 1992:5:321-25

8. Schneider RL. Evaluation of the retention of castings to endosseous dental implants. J Prothet Dent 1987;58(1):73-78.

9. Covey DA, K ent DK, St. Germain HA J r, K oka S. Effects of abutment size and luting cement type on the uniaxial retention force of implant-supported crowns. J Prosthet Dent 2000;83: 344-48.

10. M isch C. Screw-retained versus cement-retained implantsupported prosthesis. Pract Periodontics A esthet Dent 1995; 7(9):15-18.

11. M CGlumphy E. Implant-supported fixed prosthesis. In: Rosenstiel SF, Land MF, Fujimoto J. Contemporary fixed prosthodontics (4th ed). New Delhi: Elsevier 2006:397-40.

12. Koka S, Ewoldsen NO, Dana CL, B etty M W. The effect of cementing agent and technique on the retention of a CeraOne Gold Cylinder: A pilot study. Implant Dent 1995;4:32-34.

13. Singer A, Serfaty V . Cement-retained implant-supported fixed partial dentures: A 6-month to 3-year follow-up. Int J Oral M axillofac Implants 1996;11:645-49.

14. Bernal G, Okamura M, M unoz CA. The effect of taper, length and cement type on resistance to dislodgement of cementretained implant-supported restorations. J Prostho 2003; 12(2):111-15.

\section{ABOUT THE AUTHORS}

\section{Jay Dipak Matani (Corresponding Author)}

Postgraduate Student, Department of Prosthodontics, M A Rangoonwala College of Dental Sciences and Research Centre, Pune M aharashtra, India, e-mail: matanijay@gmail.com

\section{Natasha Parulekar}

Intern, Department of Prosthodontics, M A Rangoonwala College of Dental Sciences and Research Centre, Pune, M aharashtra, India

\section{Mohit Kheur}

Professor, D epartment of Prosthodontics, M A Rangoonwala College of Dental Sciences and Research Centre, Pune, M aharashtra, India

\section{Shantanu Jambhekar}

L ecturer, Department of Prosthodontics, Terna Dental College M umbai, M aharashtra, India

\section{MK Supriya}

Professor, Department of Oral Pathology, DY Patil Dental College Pune, $M$ aharashtra, India 